\title{
A NEW NUMERICAL TECHNIQUE FOR SOLVING FRACTIONAL PARTIAL DIFFERENTIAL EQUATIONS
}

\author{
OMER ACAN AND DUMITRU BALEANU
}

Received 03 April, 2017

\begin{abstract}
We propose conformable Adomian decomposition method (CADM) for fractional partial differential equations (FPDEs). This method is a new Adomian decomposition method (ADM) based on conformable derivative operator (CDO) to solve FPDEs. At the same time, conformable reduced differential transform method (CRDTM) for FPDEs is briefly given and a numerical comparison is made between this method and the newly introduced CADM. In applied science, CADM can be used as an alternative method to obtain approximate and analytical solutions for FPDEs as CRDTM. In this study, linear and non-linear three problems are solved by these two methods. In these methods, the obtained solutions take the form of a convergent series with easily computable algorithms. For the applications, the obtained results by these methods are compared to each other and with the exact solutions. When applied to FPDEs, it is seem that CADM approach produces easy, fast and reliable solutions as CRDTM.
\end{abstract}

2010 Mathematics Subject Classification: 34A08; 34K28

Keywords: numerical solution, Adomian decomposition method, reduced differential transform method, fractional derivative, conformable derivative, partial differential equations, fractional diffusion equation, fractional gas dynamical equation

\section{INTRODUCTION}

Fractional differential equations have a substantial contributions in fields,e.g. optics, biology, physics, chemistry, mathematics, fluids mechanics, applied mathematics, and engineering [18,26,40-42]. We recall that finding an analytical solutions to these problems is not always possible [9-11,20,24,27-29,37-39]. As a result, it becomes crucial to manage these problems appropriately and solve them or develop the required solutions. ADM, which is introduced [4-6] in the 1980's, is one of the important mathematical methods used to solve many problems in real world. Since then, a number of studies have been conducted on ADM such as linear and non-linear, homogeneous and non-homogeneous operator equations which including fractional or non-fractional ODEs, PDEs, integral equations, integro-differential equations, etc. (see $[12,13,15,16,25,30,32-36]$ and references therein). A new derivative called CDO was suggested $[1,7,22]$. By the help of it, the behaviors of many problems were investigated and some solutions techniques were applied 
$[1-3,7,8,14,17,19,21,23,31]$. This new subject gives academicians an opportunity to study further in many engineering, physical and applied mathematics problems.

The aim of this study is to introduce CADM by using CDO and ADM for the first time in the literature. This method can be used to solve many linear and nonlinear FPDEs. We will briefly mentioned CRDTM to compare our CADM with it. The problems will be solved both by the CRDTM and the first proposed CADM. The obtained solutions by these methods will be compared. Thus, in section 2, we present some basic definitions and important properties of CDO. Next, in section 3 , we propose CADM. In sections 4, we introduce CRDTM to compare with our method. In section 5, we give applications of CADM and CRDTM. We give the conclusion in the final section.

\section{BASIC DEFINITIONS}

Definition 1. Given a function $f_{1}:[0, \infty) \rightarrow \mathbb{R}$. Then the CDO of $f_{1}$ order $\alpha$ is defined by $[1,7,22]$ :

$$
\left(T_{\alpha} f_{1}\right)(t)=\lim _{\varepsilon \rightarrow 0} \frac{f_{1}\left(t+\varepsilon t^{1-\alpha}\right)-f_{1}(t)}{\varepsilon}
$$

for all $t>0, \alpha \in(0,1]$.

Lemma $1([1,7,22])$. Let $f_{1}, g_{1}$ be $\alpha$ and $\beta$-differentiable at a point $t>0$ for $\alpha$. Then

(i) $T_{\alpha}\left(a f_{1}+b g_{1}\right)=a\left(T_{\alpha} f_{1}\right)+b\left(T_{\alpha} g_{1}\right)$ for all $a, b \in \mathbb{R}$ and $\alpha \in(0,1]$,

(ii) $T_{\alpha}\left(f_{1}(t)\right)=0$, for constant function $f_{1}(t)=\lambda, \alpha \in(0,1]$,

(iii) $T_{\alpha}\left(f_{1} g_{1}\right)=f_{1}\left(T_{\alpha} g_{1}\right)+g_{1}\left(T_{\alpha} f_{1}\right), \alpha \in(0,1]$,

(iv) $T_{\alpha}\left(f_{1} / g_{1}\right)=\frac{g_{1}\left(T_{\alpha} f_{1}\right)-f_{1}\left(T_{\alpha} g_{1}\right)}{g_{1}^{2}}, \alpha \in(0,1]$,

(v) If $f_{1}$ is $n$ times differentiable at $t$, then $T_{\alpha}\left(f_{1}(t)\right)=t^{\lceil\alpha\rceil-\alpha} f_{1}{ }^{(\lceil\alpha\rceil)}(t), \alpha \in(n, n+$ 1]. Where $\lceil\alpha\rceil$ is the smallest integer greater than or equal to $\alpha$.

Lemma 2. [1] Suppose that $f_{1}$ is infinitely $\alpha$-differentiable function for $\alpha \in(0,1]$ at a neighborhood of a point $t_{0}$. Then $f_{1}$ has the conformable power series expansion:

$$
f_{1}(t)=\sum_{k=0}^{\infty} \frac{\left({ }_{t} T_{\alpha}^{(k)} f_{1}\right)\left(t_{0}\right)\left(t-t_{0}\right)^{\alpha k}}{\alpha^{k} k !}, t_{0}<t<t_{0}+R^{1 / \alpha}, R>0 .
$$

Here $\left(T_{\alpha}^{(k)} f_{1}\right)\left(t_{0}\right)$ denotes the application of the conformable derivative for $k$ times. 


\section{CONFORMABLE AdOMIAN DECOMPOSITION METHOD}

We will briefly introduce CADM for FPDEs in this section. We write the nonlinear FPDEs in the standard operator form

$$
L_{\alpha}(u(x, t))+R(u(x, t))+N(u(x, t))=g(x, t)
$$

where $L_{\alpha}={ }_{\alpha} T$ is a linear operator with conformable derivative of order $\alpha(n<$ $\alpha \leq n+1), N$ is a non-linear operator, $R$ is the other part of the linear operator and $g(x, t)$ is a non-homogeneous term. If the linear operator in eq. (3.1) is applied to Lemma 1, the following equation is obtained:

$$
t^{\lceil\alpha\rceil-\alpha} \frac{\partial^{\lceil\alpha\rceil}}{\partial t^{\lceil\alpha\rceil}} u(x, t)+R(u(x, t)) N(u(x, t))=g(x, t) .
$$

Applying $L_{\alpha}^{-1}=\int_{0}^{t} \int_{0}^{\xi_{1}} \cdots \int_{n}^{\xi_{n-1}} \frac{1}{\left.\xi_{n} \Gamma \alpha\right\rceil-\alpha}(). d \xi_{n} d \xi_{n-1} \cdots d \xi_{1},(n<\alpha \leq n+1)$ the inverse of operator, to both sides of (3.2), it is obtained as

$$
L_{\alpha}^{-1} L_{\alpha}(u(x, t))=L_{\alpha}^{-1} g(x, t)-L_{\alpha}^{-1} R(u(x, t))-L_{\alpha}^{-1} N(u(x, t)) .
$$

The general solution of the given equation is decomposed into the sum

$$
u(x, t)=\sum_{n=0}^{\infty} u_{n}(x, t) .
$$

The non-linear part $N(u)$ can be decomposed into the infinite polynomial series obtained by

$$
N(u)=\sum_{n=0}^{\infty} A_{n},\left(u_{0}, u_{1}, \ldots, u_{n}\right),
$$

where $A_{n}$ is the so-called Adomian polynomials (APs). These APs can be calculated for all types of non-linearity by the help of algorithms built by Adomian $[5,6,12,15$, $25,32] . u$ and $N(u)$, respectively, is obtained as

$$
u=\sum_{i=0}^{\infty} \lambda^{i} u_{i}, N(u)=N\left(\sum_{i=0}^{\infty} \lambda^{i} u_{i}\right)=\sum_{i=0}^{\infty} \lambda^{i} A_{i}
$$

where $\lambda$ is the convenience parameter. From (3.6), APs $A_{n}$ are obtained as

$$
n ! A_{n}=\frac{d^{n}}{d \lambda^{n}}\left[N\left(\sum_{n=0}^{\infty} \lambda^{n} u_{n}\right)\right]_{\lambda=0} .
$$

These APs can be calculated easily with the following Maple code:

Substituting (3.4) and (3.5) into (3.3), it is obtained

$$
\sum_{n=0}^{\infty} u_{n}=\theta+L_{\alpha}^{-1} g-L_{\alpha}^{-1} R\left(\sum_{n=0}^{\infty} u_{n}\right)-L_{\alpha}^{-1}\left(\sum_{n=0}^{\infty} A_{n}\right) .
$$




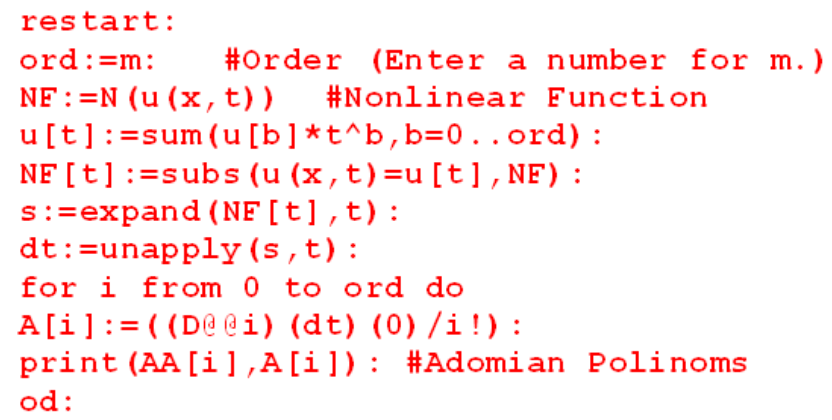

where $\theta=u(x, 0)$ is initial condition (IC). From (3.7), the iterates are defined by the following recursive formulas:

$$
\begin{gathered}
u_{0}=\theta+L_{\alpha}^{-1} g, \\
u_{1}=-L_{\alpha}^{-1} R u_{0}-L_{\alpha}^{-1} A_{0}, \\
\vdots \\
u_{n+1}=-L_{\alpha}^{-1} R u_{n}-L_{\alpha}^{-1} A_{n}, n \geq 0 .
\end{gathered}
$$

Therefore, from (3.8), the approximate solution of (3.1) is obtained by

$$
\tilde{u}_{m}(x, t)=\sum_{n=0}^{m} u_{n}(x, t) .
$$

Hence, from (3.9), the exact solution of (3.1) can be obtained as

$$
u(x, t)=\lim _{m \rightarrow \infty} \tilde{u}_{m}(x, t) .
$$

\section{CONFORMABLE REDUCED DifFERENTIAL TRANSFORM METHOD}

In this section, it is given basic definitions and properties of CRDTM for FPDEs [3].

Definition 2. Assume $u(x, t)$ is analytic function and differentiated continuously with respect to time $t$ and space $x$ in the its domain. the conformable reduced differential transformed (CRDT) of $u(x, t)$ is defined as [3]

$$
U_{k}^{\alpha}(x)=\frac{1}{\alpha^{k} k !}\left[\left({ }_{t} T_{\alpha}^{(k)} u\right)\right]_{t=t_{0}}
$$

where some $0<\alpha \leq 1, \alpha$ is describing the order of CDO, ${ }_{t} T_{\alpha}^{(k)} u=\underbrace{\left({ }_{t} T_{\alpha t} T_{\alpha} \cdots{ }_{t} T_{\alpha}\right)}_{k \text { times }} u(x, t)$ and $U_{k}^{\alpha}(x)$ is the CRDT function. 
Definition 3. Let $U_{k}^{\alpha}(x)$ be the CRDT of $u(x, t)$. Inverse CRDT of $U_{k}^{\alpha}(x)$ is defined as [3]

$$
u(x, t)=\sum_{k=0}^{\infty} U_{k}^{\alpha}(x)\left(t-t_{0}\right)^{\alpha k}=\sum_{k=0}^{\infty} \frac{1}{\alpha^{k} k !}\left[{ }_{t} T_{\alpha}^{(k)} u\right]_{t=t_{0}}\left(t-t_{0}\right)^{\alpha k}
$$

CRDT of ICs for integer order derivatives are defined as [3]

$$
U_{k}^{\alpha}(x)=\left\{\begin{array}{ll}
\frac{1}{(\alpha k) !}\left[\frac{\partial^{\alpha k}}{\partial t^{\alpha k}} u(x, t)\right]_{t=t_{0}} & \text { if } \alpha k \in \mathbb{Z}^{+} \\
0 & \text { if } \alpha k \notin \mathbb{Z}^{+}
\end{array} \quad \text { for } k=0,1,2, \ldots,\left(\frac{n}{\alpha}-1\right)\right.
$$

where $n$ is the order of CDO of PDE.

By consideration of

$$
U_{0}^{\alpha}(x)=f(x)
$$

as transformation of IC

$$
u(x, 0)=f(x) .
$$

A straightforward iterative calculations gives the $U_{k}^{\alpha}(x)$ values for $k=1,2,3, \ldots, n$. Then the set of $\left\{U_{k}^{\alpha}(x)\right\}_{k=0}^{n}$ gives the approximate result as:

$$
\tilde{u}_{n}(x, t)=\sum_{k=0}^{n} U_{k}^{\alpha}(x) t^{k \alpha},
$$

where $n$ is approximate result order. The exact solution can be obtained as:

$$
u(x, t)=\lim _{n \rightarrow \infty} \tilde{u}_{n}(x, t)
$$

The fundamental operations of CRDTM that can be deduced from Definition 2 and Definition 3 are listed in Table 1 [3].

\section{NUMERICAL CONSIDERATION}

To illustrate the effectiveness of the given CADM and CRDTM, three examples are considered in this section. All the results are calculated by software MAPLE.

Example 1. Firstly, consider the linear time and space fractional diffusion equation:

with the IC

$$
\frac{\partial^{\alpha}}{\partial t^{\alpha}} u(x, t)=\frac{\partial^{2 \beta}}{\partial x^{2 \beta}} u(x, t) t>0, x \in R, 0<\alpha, \beta \leq 1
$$

$$
u(x, 0)=\sin \left(\frac{x^{\beta}}{\beta}\right) .
$$

Exact result of the problem (5.1) in conformable sense is

$$
u(x, t)=\sin \left(\frac{x^{\beta}}{\beta}\right) e^{-\frac{t^{\alpha}}{\alpha}} .
$$


TABLE 1. Basic operations of the CRDTM [3].

\begin{tabular}{ll}
\hline Original function & Transformed function \\
\hline$u(x, t)$ & $U_{k}^{\alpha}(x)=\frac{1}{\alpha^{k} k !}\left[\left({ }_{t} T_{\alpha}^{(k)} u\right)\right]_{t=t_{0}}$ \\
$u(x, t)=a v(x, t) \pm b w(x, t)$ & $U_{k}^{\alpha}(x)=a V_{k}^{\alpha}(x) \pm b W_{k}^{\alpha}(x)$ \\
$u(x, t)=v(x, t) w(x, t)$ & $U_{k}^{\alpha}(x)=\sum_{s=0}^{k} V_{s}^{\alpha}(x) W_{k-s}^{\alpha}(x)$ \\
$u(x, t)={ }_{t} T_{\alpha} v(x, t)$ & $U_{k}^{\alpha}(x)=\alpha(k+1) V_{k+1}^{\alpha}(x)$ \\
$u(x, t)=x^{m}\left(t-t_{0}\right)^{n}$ & $U_{k}^{\alpha}(x)=x^{m} \delta\left(k-\frac{n}{\alpha}\right)$ \\
\hline
\end{tabular}

Solution by CADM: Solve this problem by using CADM. Let $L_{\alpha}=T_{\alpha}=\frac{\partial^{\alpha}}{\partial t^{\alpha}}$ be a linear operator, then the operator form of (5.1) is as follows

$$
T_{\alpha} u(x, t)=\frac{\partial^{2 \beta} u(x, t)}{\partial x^{2 \beta}} t>0, x \in R, 0<\alpha, \beta \leq 1
$$

By the help of Lemma 1, eq. (5.3) can be written as

$$
t^{1-\beta} \frac{\partial u(x, t)}{\partial t}=\frac{\partial^{2 \beta} u(x, t)}{\partial x^{2 \beta}} t>0, x \in R, 0<\alpha, \beta \leq 1 .
$$

If $L_{\alpha}^{-1}=\int_{0}^{t} \frac{1}{\xi^{1-\alpha}}(). d \xi$, which is the inverse of $L_{\alpha}$, is applied to both sides of eq. (5.4), we get

$$
u(x, t)=u(x, 0)-L_{\alpha}^{-1}\left(\frac{\partial^{2 \beta}}{\partial x^{2 \beta}} u(x, t)\right) .
$$

According to (3.8) and the IC (5.2), we can write

$$
\begin{gathered}
u_{0}=\sin \left(\frac{x^{\beta}}{\beta}\right), \\
u_{1}=-L_{\alpha}^{-1}\left(\frac{\partial^{2}}{\partial x^{2}} u_{0}\right), \\
\vdots \\
u_{n+1}=-L_{\alpha}^{-1}\left(\frac{\partial^{2}}{\partial x^{2}} u_{n}\right), n \geq 0 .
\end{gathered}
$$


From (5.5), we conclude the terms of decomposition series as:

$$
\begin{aligned}
& u_{0}=\sin \left(\frac{x^{\beta}}{\beta}\right), u_{1}=-\sin \left(\frac{x^{\beta}}{\beta}\right) \frac{t^{\alpha}}{\alpha} \\
& u_{2}=\sin \left(\frac{x^{\beta}}{\beta}\right) \frac{t^{2 \alpha}}{2 \alpha^{2}}, \cdots, u_{n}=(-1)^{n} \sin \left(\frac{x^{\beta}}{\beta}\right) \frac{t^{n \alpha}}{n ! \alpha^{n}}, \cdots
\end{aligned}
$$

Thus, by using (5.6), the approximate solution of (5.1) obtained by CADM is

$$
\tilde{u}_{m}(x, t)=\sum_{n=0}^{m} u_{n}(x, t)=\sum_{n=0}^{m}(-1)^{n} \sin \left(\frac{x^{\beta}}{\beta}\right) \frac{t^{n \alpha}}{n ! \alpha^{n}} .
$$

From (5.7) we obtain

$$
u(x, t)=\lim _{m \rightarrow \infty} \tilde{u}_{m}(x, t)=\sin \left(\frac{x^{\beta}}{\beta}\right) e^{-\frac{t^{\alpha}}{\alpha}} .
$$

This analytical approximate solution (5.8) is the exact solution.

Solution by CRDTM: Now solve this problem by using CRDTM. By taking the CRDT of (5.1), it can be obtained that

$$
\alpha(k+1) U_{k+1}^{\alpha}(x)=\frac{\partial^{2 \beta}}{\partial x^{2 \beta}} U_{k}^{\alpha}(x)
$$

where $U_{k}^{\alpha}(x)$ is the CRDT function. From the IC (5.2) we write

$$
U_{0}^{\alpha}(x)=\sin \left(\frac{x^{\beta}}{\beta}\right)
$$

Substituting (5.10) into (5.9), it can be obtained the following $U_{k}^{\alpha}(x)$ values

$$
\begin{aligned}
& U_{1}^{\alpha}(x)=-\sin \left(\frac{x^{\beta}}{\beta}\right) \frac{1}{\alpha}, U_{2}^{\alpha}(x)=\sin \left(\frac{x^{\beta}}{\beta}\right) \frac{1}{2 ! \alpha^{2}}, \\
& U_{3}^{\alpha}(x)=-\sin \left(\frac{x^{\beta}}{\beta}\right) \frac{1}{3 ! \alpha^{3}}, \cdots, U_{n}^{\alpha}(x)=\sin \left(\frac{x^{\beta}}{\beta}\right) \frac{(-1)^{n}}{n ! \alpha^{n}}, \cdots
\end{aligned}
$$

Then, the set of values $\left\{U_{k}^{\alpha}(x)\right\}_{k=0}^{n}$ gives the following approximate result

$$
\tilde{u}_{n}(x, t)=\sum_{k=0}^{n} U_{k}^{\alpha}(x) t^{k \alpha}=\sum_{k=0}^{n} \sin \left(\frac{x^{\beta}}{\beta}\right) \frac{(-1)^{k}}{k ! \alpha^{k}} t^{k \alpha} .
$$

From (5.11) we obtain

$$
u(x, t)=\lim _{n \rightarrow \infty} \tilde{u}_{n}(x, t)=\sin \left(\frac{x^{\beta}}{\beta}\right) e^{-\frac{t^{\alpha}}{\alpha}} .
$$


This approximate solution (5.12) is the exact solution.

Remark 1. If take $\alpha=\beta=1$ in the problem (5.1), then Example 1 is reduced to standard diffusion equation

with IC

$$
\frac{\partial}{\partial t} u(x, t)=\frac{\partial^{2}}{\partial x^{2}} u(x, t) t>0, x \in R
$$

$$
u(x, 0)=\sin (x)
$$

and our analytical approximate solutions (5.8) and (5.12) imply

$$
u(x, t)=\sin (x) e^{-t}
$$

and this solutions is the exact result of the standard problem in the literature.

The Aproximate solutions obtained by both CADM and CRDTM give us the exact solution.

Example 2. Secondly, let us consider the non-linear time and space fractional gas dynamics equation:

$$
\frac{\partial^{\alpha}}{\partial t^{\alpha}} u(x, t)+\frac{1}{2} \frac{\partial^{\beta}}{\partial x^{\beta}} u^{2}(x, t)-u(x, t)(1-u(x, t))=0,0 \alpha, \beta \leq 1
$$

subject to IC

$$
u(x, 0)=e^{-\frac{x^{\beta}}{\beta}} .
$$

The exact solutions of (5.13) in conformable sense is

$$
u(x, t)=e^{\frac{t^{\alpha}}{\alpha}-\frac{x^{\beta}}{\beta}} .
$$

Solution by CADM: Solve the problem by using CADM. Let $L_{\alpha}=T_{\alpha}=\frac{\partial^{\alpha}}{\partial t^{\alpha}}$ be a linear operator, then the operator form of (5.13) is as follows

$$
T_{\alpha} u(x, t)=-\frac{1}{2} \frac{\partial^{\beta}}{\partial x^{\beta}} u^{2}(x, t)+u(x, t)(1-u(x, t)), 0 \alpha, \beta \leq 1 .
$$

By the help of Lemma 1, eq. (5.15) can be written as

$$
t^{1-\alpha} \frac{\partial u(x, t)}{\partial t}=u(x, t)-u(x, t) \frac{\partial^{\beta}}{\partial x^{\beta}} u(x, t)-u^{2}(x, t), 0 \alpha, \beta \leq 1 .
$$

If $L_{\alpha}^{-1}=\int_{0}^{t} \frac{1}{\xi^{1-\alpha}}(). d \xi$, which is the inverse of $L_{\alpha}$, is applied to both sides of eq. (5.16), we get

$$
u(x, t)=u(x, 0)+L_{\alpha}^{-1}(u(x, t))-L_{\alpha}^{-1}\left(u(x, t) \frac{\partial^{\beta}}{\partial x^{\beta}} u(x, t)+u^{2}(x, t)\right) .
$$


According to (3.8) and IC (5.14), we can write the following recursive relations:

$$
\begin{gathered}
u_{0}=e^{-\frac{x^{\beta}}{\beta}} \\
u_{1}=L_{\alpha}^{-1}\left(u_{0}\right)-L_{\alpha}^{-1}\left(A_{0}\right) \\
\vdots \\
u_{n+1}=L_{\alpha}^{-1}\left(u_{n}\right)-L_{\alpha}^{-1}\left(A_{n}\right), n \geq 0 .
\end{gathered}
$$

where $A_{n}$ 's are APs. By using the Maple code above, for the non-linear term

$$
N(u(x))=u(x, t) \frac{\partial}{\partial x} u(x, t)+u^{2}(x, t),
$$

the APs can be obtain as:

$$
\begin{gathered}
A_{0}=u_{0}^{2}+u_{0} \frac{\partial^{\beta}}{\partial x^{\beta}} u_{0} \\
A_{1}=2 u_{0} u_{1}+u_{0} \frac{\partial^{\beta}}{\partial x^{\beta}} u_{1}+u_{1} \frac{\partial^{\beta}}{\partial x^{\beta}} u_{0} \\
A_{2}=u_{1}^{2}+2 u_{0} u_{2}+u_{0} \frac{\partial^{\beta}}{\partial x^{\beta}} u_{2}+u_{1} \frac{\partial^{\beta}}{\partial x^{\beta}} u_{1}+u_{2} \frac{\partial^{\beta}}{\partial x^{\beta}} u_{0} \\
A_{3}=2 u_{1} u_{2}+2 u_{0} u_{3}+u_{0} \frac{\partial^{\beta}}{\partial x^{\beta}} u_{3}+u_{1} \frac{\partial^{\beta}}{\partial x^{\beta}} u_{2}+u_{2} \frac{\partial^{\beta}}{\partial x^{\beta}} u_{1}+u_{3} \frac{\partial^{\beta}}{\partial x^{\beta}} u_{0}
\end{gathered}
$$

From (5.17) and (5.18), we conclude the terms of decomposition series as:

$$
u_{0}=e^{-\frac{x^{\beta}}{\beta}}, u_{1}=e^{-\frac{x^{\beta} \beta}{\beta}} \frac{t^{\alpha}}{\alpha}, u_{2}=e^{-\frac{x^{\beta}}{\beta}} \frac{t^{2 \alpha}}{2 \alpha^{2}}, \cdots, u_{n}=e^{-\frac{x^{\beta} \beta}{\beta}} \frac{t^{n \alpha}}{n ! \alpha^{n}}, \cdots
$$

Thus, From (5.19), the approximate solution of (5.13) obtained by CADM is

$$
\tilde{u}_{m}(x, t)=\sum_{n=0}^{m} u_{n}(x, t)=\sum_{n=0}^{m} e^{-\frac{x^{\beta}}{\beta}} \frac{t^{n \alpha}}{n ! \alpha^{n}} .
$$

From (5.20) we obtain

$$
u(x, t)=\lim _{m \rightarrow \infty} \tilde{u}_{m}(x, t)=e^{\frac{t^{\alpha}}{\alpha}-\frac{x^{\beta}}{\beta}} .
$$

This analytical approximate solution (5.21) is the exact solution.

Solution by CRDTM: Now solve this problem by using CRDTM. By taking the CRDT of (5.13), it can be obtained that

$$
\alpha(k+1) U_{k+1}^{\alpha}(x)=-\sum_{r=0}^{k} U_{k-r}^{\alpha}(x) \frac{\partial^{\beta}}{\partial x^{\beta}} U_{r}^{\alpha}(x)+U_{k}^{\alpha}(x)-\sum_{r=0}^{k} U_{k-r}^{\alpha}(x) U_{r}^{\alpha}(x)
$$

where $U_{k}^{\alpha}(x)$ is the CRDT function. From the IC (5.14) we write

$$
U_{0}^{\alpha}(x)=e^{-\frac{x^{\beta}}{\beta}}
$$


Substituting (5.23) into (5.22), it can be obtained the following $U_{k}^{\alpha}(x)$ values

$$
U_{1}^{\alpha}(x)=e^{-\frac{x^{\beta}}{\beta}} \frac{1}{\alpha}, U_{2}^{\alpha}(x)=e^{-\frac{x^{\beta}}{\beta}} \frac{1}{2 ! \alpha^{2}}, \cdots, U_{n}^{\alpha}(x)=e^{-\frac{x^{\beta}}{\beta}} \frac{1}{n ! \alpha^{n}}, \cdots
$$

Then, the set of values $\left\{U_{k}^{\alpha}(x)\right\}_{k=0}^{n}$ gives the following approximate result

$$
\tilde{u}_{n}(x, t)=\sum_{k=0}^{n} U_{k}^{\alpha}(x) t^{k \alpha}=\sum_{k=0}^{n} e^{-\frac{x^{\beta}}{\beta}} \frac{1}{k ! \alpha^{k}} t^{k \alpha} .
$$

From (5.24) we obtain

$$
u(x, t)=\lim _{n \rightarrow \infty} \tilde{u}_{n}(x, t)=e^{\frac{t^{\alpha}}{\alpha}-\frac{x^{\beta}}{\beta}} .
$$

This approximate solution (5.25) is the exact solution.

Remark 2. If take $\alpha=\beta=1$ in the problem (5.13), then Example 2 is reduced to standard gas dynamics equation

with IC

$$
\frac{\partial}{\partial t} u(x, t)+\frac{1}{2} \frac{\partial}{\partial x} u^{2}(x, t)-u(x, t)(1-u(x, t))=0
$$

$$
u(x, 0)=e^{-x}
$$

our analytical approximate solutions (5.25) and (5.21) imply

$$
u(x, t)=e^{t-x}
$$

and this solution is the exact result of the standard problem in the literature.

The approximate solutions obtained by both CADM and CRDTM give us the existed exact solution.

Example 3. Finally, let us consider the non-linear time and space FPDE:

subject to IC

$$
\frac{\partial^{\alpha}}{\partial t^{\alpha}} u(x, t)+(1+u(x, t)) \frac{\partial^{\alpha}}{\partial x^{\alpha}} u(x, t)=0,0 \alpha \leq 1
$$

$$
u(x, 0)=\frac{x^{\alpha}-\alpha}{2 \alpha} .
$$

The exact solutions of (5.26) in conformable sense is

$$
u(x, t)=\frac{x^{\alpha}-t^{\alpha}-\alpha}{t^{\alpha}-2 \alpha} .
$$

Solution by CADM: Solve the problem by using CADM. Let $L_{\alpha}=T_{\alpha}=\frac{\partial^{\alpha}}{\partial t^{\alpha}}$ be a linear operator, then the operator form of (5.26) is as follows

$$
T_{\alpha} u(x, t)=-(1+u(x, t)) \frac{\partial^{\alpha}}{\partial x^{\alpha}} u(x, t), 0 \alpha \leq 1 .
$$


By the help of Lemma 1, eq. (5.28) can be written as

$$
t^{1-\alpha} \frac{\partial u(x, t)}{\partial t}=-\frac{\partial^{\alpha}}{\partial x^{\alpha}} u(x, t)-u(x, t) \frac{\partial^{\alpha}}{\partial x^{\alpha}} u(x, t), 0 \alpha \leq 1 .
$$

If $L_{\alpha}^{-1}=\int_{0}^{t} \frac{1}{\xi^{1-\alpha}}(). d \xi$, which is the inverse of $L_{\alpha}$, is applied to both sides of eq. (5.29), we get

$$
u(x, t)=u(x, 0)-L_{\alpha}^{-1}\left(\frac{\partial^{\alpha}}{\partial x^{\alpha}} u(x, t)\right)-L_{\alpha}^{-1}\left(u(x, t) \frac{\partial^{\alpha}}{\partial x^{\alpha}} u(x, t)\right) .
$$

According to 3.8 and IC (5.27), we can write the following recursive relations:

$$
\begin{gathered}
u_{0}=\frac{x^{\alpha}-\alpha}{2 \alpha} \\
u_{1}=L_{\alpha}^{-1}\left(u_{0}\right)-L_{\alpha}^{-1}\left(A_{0}\right) \\
\vdots \\
u_{n+1}=L_{\alpha}^{-1}\left(u_{n}\right)-L_{\alpha}^{-1}\left(A_{n}\right), n \geq 0 .
\end{gathered}
$$

where $A_{n}$ 's are APs. By using the Maple code above, for the non-linear term

$$
N(u(x))=u(x, t) \frac{\partial}{\partial x} u(x, t)+u^{2}(x, t),
$$

the APs can be obtain as:

$$
\begin{gathered}
A_{0}=u_{0} \frac{\partial^{\alpha}}{\partial x^{\alpha}} u_{0} \\
A_{1}=u_{0} \frac{\partial^{\alpha}}{\partial x^{\alpha}} u_{1}+u_{1} \frac{\partial^{\alpha}}{\partial x^{\alpha}} u_{0} \\
A_{2}=u_{0} \frac{\partial^{\alpha}}{\partial x^{\alpha}} u_{2}+u_{1} \frac{\partial^{\alpha}}{\partial x^{\alpha}} u_{1}+u_{2} \frac{\partial^{\alpha}}{\partial x^{\alpha}} u_{0} \\
A_{3}=u_{0} \frac{\partial^{\alpha}}{\partial x^{\alpha}} u_{3}+u_{1} \frac{\partial^{\alpha}}{\partial x^{\alpha}} u_{2}+u_{2} \frac{\partial^{\alpha}}{\partial x^{\alpha}} u_{1}+u_{3} \frac{\partial^{\alpha}}{\partial x^{\alpha}} u_{0}
\end{gathered}
$$

From (5.30) and (5.31), we conclude the terms of decomposition series as:

$$
\begin{aligned}
& u_{0}=\frac{x^{\alpha}-\alpha}{2 \alpha}, u_{1}=-\frac{x^{\alpha}+\alpha}{(2 \alpha)^{2}} t^{\alpha}, u_{2}=\frac{x^{\alpha}+\alpha}{(2 \alpha)^{3}} t^{2 \alpha}, \\
& u_{3}=-\frac{x^{\alpha}+\alpha}{(2 \alpha)^{4}} t^{3 \alpha}, \cdots, u_{n}=(-1)^{n} \frac{x^{\alpha}+\alpha}{(2 \alpha)^{n+1}} t^{n \alpha}, \cdots
\end{aligned}
$$

Thus, from (5.32), the approximate solution of (5.26) obtained by CADM is

$$
\tilde{u}_{m}(x, t)=\sum_{n=0}^{m} u_{n}(x, t)=\frac{x^{\alpha}-\alpha}{2 \alpha}+\sum_{n=1}^{m}(-1)^{m} \frac{x^{\alpha}+\alpha}{(2 \alpha)^{m+1}} t^{m \alpha} .
$$


Solution by CRDTM: Now solve this problem by using CRDTM. By taking the CRDT of (5.26), it can be obtained that

$$
\alpha(k+1) U_{k+1}(x)=-U_{k}(x)-\sum_{r=0}^{k} U_{k-r}(x) \frac{\partial}{\partial x} U_{r}(x)
$$

where $U_{k}^{\alpha}(x)$ is the CRDT function. From the IC (5.27) we write

$$
U_{0}^{\alpha}(x)=\frac{x^{\alpha}-\alpha}{2 \alpha}
$$

Substituting (5.35) into (5.34), it can be obtained the following $U_{k}^{\alpha}(x)$ values

$$
U_{1}^{\alpha}(x)=-\frac{x^{\alpha}+\alpha}{(2 \alpha)^{2}}, U_{2}^{\alpha}(x)=\frac{x^{\alpha}+\alpha}{(2 \alpha)^{3}}, \cdots, U_{n}^{\alpha}(x)=(-1)^{n} \frac{x^{\alpha}+\alpha}{(2 \alpha)^{n+1}}, \cdots
$$

Then, the set of values $\left\{U_{k}^{\alpha}(x)\right\}_{k=0}^{n}$ gives the following approximate result

$$
\tilde{u}_{m}(x, t)=\sum_{k=0}^{m} U_{k}^{\alpha}(x) t^{k \alpha}=\frac{x^{\alpha}-\alpha}{2 \alpha}+\sum_{n=1}^{m}(-1)^{m} \frac{x^{\alpha}+\alpha}{(2 \alpha)^{m+1}} t^{m \alpha} .
$$

Now, we compare the seventh iteration CADM and CRDTM solutions with the exact solution on the graphs for some $\alpha$ values. These comparisons can be seen in fig. 1 and fig. 2.
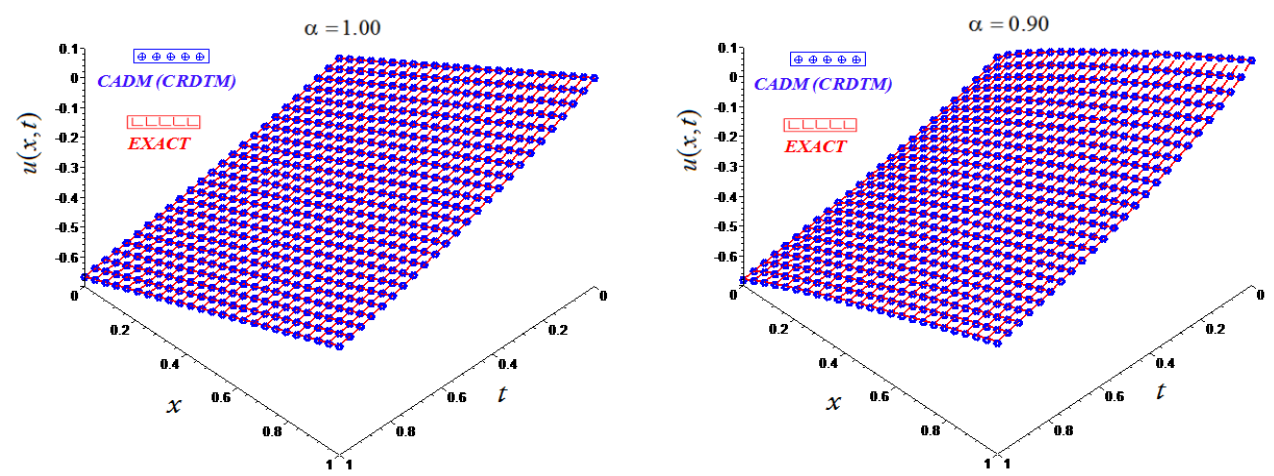

FIGURE 1. Comparison of seventh iteration approximate results of CADM (CRDTM) with the exact solutions for eq. (5.26). 

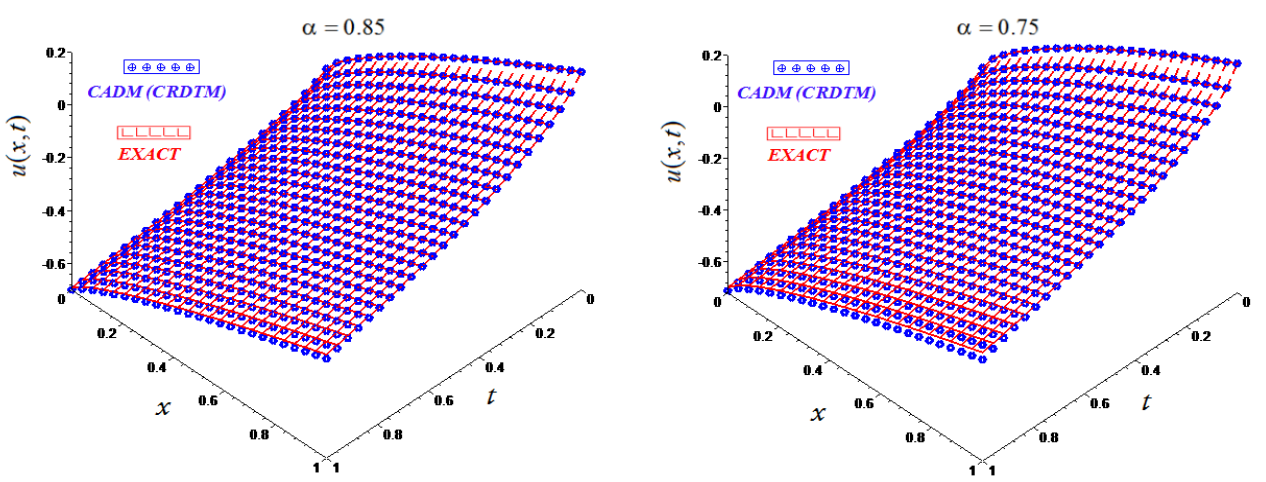

FIGURE 2. Comparison of seventh iteration approximate results of CADM (CRDTM) with the exact solutions for eq. (5.26).

\section{CONCLUSiOn}

The fundamental goal of this article is to construct the approximate solutions of FPDEs. The goal has been achieved by using CADM for the first time and it is compared with CRDTM. CADM and CRDTM are applied to different linear and non-linear conformable time and space FPDEs. And also the approximate analytical solutions obtained by CADM and CRDTM are compared to each other and with the exact solutions. CADM and CRDTM offer solutions with easily computable components as convergent series. Approximate solutions obtained by CADM are exactly same as the solutions obtained by CRDTM for time and space FPDEs. The CADM gives quantitatively reliable results as CRDTM, and also it requires less computational work than existing other methods. As a result, in recent years, FDEs emerging as models in fields such as mathematics, physics, chemistry, biology and engineering makes it necessary to investigate the methods of solutions and we hope that this study is an improvement in this direction.

\section{REFERENCES}

[1] T. Abdeljawad, "On conformable fractional calculus," Journal of computational and Applied Mathematics, vol. 279, pp. 57-66, 2015, doi: 10.1016/j.cam.2014.10.016.

[2] O. Acan, O. Firat, Y. Keskin, and G. Oturanc, "Conformable variational iteration method," New Trends in Mathematical Sciences, vol. 5, p. 172-178, 2017, doi: 10.20852/ntmsci.2017.135.

[3] O. Acan, O. Firat, Y. Keskin, and G. Oturanc, "Solution of conformable fractional partial differential equations by reduced differential transform method," Selcuk Journal of Applied Mathematics, vol. 128, p. acceped, 2016.

[4] G. Adomian, Stochastic systems. Academic Press Inc., New York, NY, USA, 1983.

[5] G. Adomian, "A review of the decomposition method in applied mathematics," Journal of mathematical analysis and applications, vol. 135, no. 2, pp. 501-544, 1988, doi: 10.1016/0022247X(88)90170-9. 
[6] G. Adomian, Solving frontier problems of physics: the decomposition method. Springer Science \& Business Media, 2013, vol. 60.

[7] D. R. Anderson and D. J. Ulness, "Newly defined conformable derivatives," Advances in Dynamical Systems and Applications, vol. 10, no. 2, pp. 109-137, 2015.

[8] A. Atangana, D. Baleanu, and A. Alsaedi, "New properties of conformable derivative," Open Mathematics, vol. 13, no. 1, pp. 1-10, 2015, doi: 10.1515/math-2015-0081.

[9] F. Atici and P. Eloe, "Initial value problems in discrete fractional calculus," Proceedings of the American Mathematical Society, vol. 137, no. 3, pp. 981-989, 2009, doi: 10.1090/S0002-993908-09626-3.

[10] D. Baleanu, K. Diethelm, E. Scalas, and J. J. Trujillo, Fractional calculus: models and numerical methods. World Scientific, 2016, vol. 5.

[11] D. Baleanu, Z. B. Guvenc, and J. A. T. Machado, New trends in nanotechnology and fractional calculus applications. Netherlands, Dordrecht: Springer, 2010.

[12] N. Bildik and A. Konuralp, "The use of variational iteration method, differential transform method and adomian decomposition method for solving different types of nonlinear partial differential equations," International Journal of Nonlinear Sciences and Numerical Simulation, vol. 7, no. 1, pp. 65-70, 2006, doi: 10.1515/IJNSNS.2006.7.1.65.

[13] B. Boutarfa, A. Akgul, and M. Inc, "New approach for the fornberg-whitham type equations," Journal of Computational and Applied Mathematics, vol. 312, pp. 13-26, 2017, doi: 10.1016/j.cam.2015.09.016.

[14] W. S. Chung, "Fractional newton mechanics with conformable fractional derivative," Journal of Computational and Applied Mathematics, vol. 290, pp. 150-158, 2015, doi: 10.1016/j.cam.2015.04.049.

[15] J.-S. Duan, R. Rach, D. Baleanu, and A.-M. Wazwaz, "A review of the adomian decomposition method and its applications to fractional differential equations," Communications in Fractional Calculus, vol. 3, no. 2, pp. 73-99, 2012.

[16] J.-S. Duan, R. Rach, and A.-M. Wazwaz, "Steady-state concentrations of carbon dioxide absorbed into phenyl glycidyl ether solutions by the adomian decomposition method," Journal of Mathematical Chemistry, vol. 53, no. 4, pp. 1054-1067, 2015, doi: 10.1007/s10910-014-0469-z.

[17] M. Ekici, M. Mirzazadeh, M. Eslami, Q. Zhou, S. P. Moshokoa, A. Biswas, and M. Belic, "Optical soliton perturbation with fractional-temporal evolution by first integral method with conformable fractional derivatives," Optik-International Journal for Light and Electron Optics, vol. 127, no. 22, pp. 10659-10669, 2016, doi: 10.1016/j.ijleo.2016.08.076.

[18] F. Gao, X.-J. Yang, and M.-D. Syed, "On linear viscoelasticity within general fractional derivatives without singular kernel," Thermal Science, vol. 21(1), pp. 335-342, 2017, doi: 10.2298/TSCI170308197G.

[19] A. Gokdogan, E. Unal, and E. Celik, "Existence and uniqueness theorems for sequential linear conformable fractional differential equations," Miskolc Mathematical Notes, vol. 17, pp. 267-279, 2016, doi: 10.18514/MMN.2016.1635.

[20] H. L. Gray and N. F. Zhang, "On a new definition of the fractional difference," Mathematics of Computation, vol. 50, no. 182, pp. 513-529, 1988, doi: 10.1090/S0025-5718-1988-0929549-2.

[21] H. Karayer, D. Demirhan, and F. Buyukkilic, "Conformable fractional nikiforov-uvarov method," Communications in Theoretical Physics, vol. 66, no. 1, pp. 12-18, 2016, doi: 10.1088/0253-6102/66/1/012.

[22] R. Khalil, M. Al-Horani, A. Yousef, and M. Sababheh, "A new definition of fractional derivative," Journal of Computational and Applied Mathematics, vol. 264, pp. 65-70, 2014, doi: 10.1016/j.cam.2014.01.002.

[23] A. Kurt, Y. Cenesiz, and O. Tasbozan, "On the solution of burgers' equation with the new fractional derivative," Open Phys, vol. 13, pp. 355-360, 2015, doi: 10.1515/phys-2015-0045. 
[24] K. S. Miller and B. Ross, An introduction to the fractional calculus and fractional differential equations. Wiley-Interscience, 1993.

[25] S. Momani and Z. Odibat, "Analytical solution of a time-fractional navier-stokes equation by adomian decomposition method," Applied Mathematics and Computation, vol. 177, no. 2, pp. 488-494, 2006, doi: 10.1016/j.amc.2005.11.025.

[26] Z. Navickas, T. Tadas, M. Romas, and R. Minvydas, "Operator-based approach for the construction of analytical soliton solutions to nonlinear fractional-order differential equations," Chaos, Solitons \& Fractals, vol. 104, pp. 625-634, 2017, doi: 10.1016/j.chaos.2017.09.026.

[27] K. Oldham and J. Spanier, The fractional calculus theory and applications of differentiation and integration to arbitrary order. Academic Press, 1974, vol. 111.

[28] I. Podlubny, Fractional Differential Equations: An Introduction to Fractional Derivatives, Fractional Differential Equations, to Methods of Their Solution and Some of Their Applications. Academic Press, 1998, vol. 198.

[29] S. G. Samko, A. A. Kilbas, and O. I. Marichev, Fractional integrals and derivatives. Gordon and Breach Science Publishers, Yverdon, 1993.

[30] M. Turkyilmazoglu, "Determination of the correct range of physical parameters in the approximate analytical solutions of nonlinear equations using the adomian decomposition method," Mediterranean Journal of Mathematics, vol. 13, no. 6, pp. 4019-4037, 2016, doi: 10.1007/s00009-0160730-8.

[31] E. Unal and A. Gokdogan, "Solution of conformable fractional ordinary differential equations via differential transform method," Optik-International Journal for Light and Electron Optics, vol. 128, pp. 264-273, 2017, doi: 10.1016/j.ijleo.2016.10.031.

[32] Q. Wang, "Numerical solutions for fractional kdv-burgers equation by adomian decomposition method," Applied Mathematics and Computation, vol. 182, no. 2, pp. 1048-1055, 2006, doi: 10.1016/j.amc.2006.05.004.

[33] A.-M. Wazwaz, "Adomian decomposition method for a reliable treatment of the bratu-type equations," Applied Mathematics and Computation, vol. 166, no. 3, pp. 652-663, 2005, doi: 10.1016/j.amc.2004.06.059.

[34] A.-M. Wazwaz, "Adomian decomposition method for a reliable treatment of the emden-fowler equation," Applied Mathematics and Computation, vol. 161, no. 2, pp. 543-560, 2005, doi: 10.1016/j.amc.2003.12.048.

[35] A.-M. Wazwaz, R. Rach, and L. Bougoffa, "Dual solutions for nonlinear boundary value problems by the adomian decomposition method," International Journal of Numerical Methods for Heat \& Fluid Flow, vol. 26, no. 8, pp. 2393-2409, 2016, doi: 10.1108/HFF-10-2015-0439.

[36] A.-M. Wazwaz, R. Rach, L. Bougoffa, and J.-S. Duan, "Solving the lane-emden-fowler type equations of higher orders by the adomian decomposition method," Comput. Model. Eng. Sci.(CMES), vol. 100, no. 6, pp. 507-529, 2014

[37] X.-J. Yang, Local fractional functional analysis and its applications. Hong Kong: Asian Academic Publisher Limited, 2011.

[38] X.-J. Yang, Advanced local fractional calculus and its applications. World Science, New York, USA, 2012.

[39] X.-J. Yang, D. Baleanu, and H. M. Srivastava, Local fractional integral transforms and their applications. Academic Press, 2015.

[40] X.-J. Yang, F. Gao, and H. M. Srivastava, "New rheological models within local fractional derivative," Romanian Reports in Physics, vol. 69(3), p. 113, 2017.

[41] X.-J. Yang, F. Gao, and H. M. Srivastava, "On a fractal lc-electric circuit modeled by local fractional calculus," Communications in Nonlinear Science and Numerical Simulation, vol. 47, pp. 200-206, 2017, doi: 10.1016/j.cnsns.2016.11.017. 
[42] X.-J. Yang, J. A. T. Machado, and D. Baleanu, "Anomalous diffusion models with general fractional derivatives within the kernels of the extended mittag-leffler type functions," Romanian Reports in Physics, vol. 69, p. 115, 2017.

\section{Authors' addresses}

\section{Omer Acan}

Siirt University, Department of Mathematics, Art and Science Faculty, 56100 Siirt, Turkey

E-mail address: omeracan@yahoo.com

\section{Dumitru Baleanu}

Institute of Space Sciences, Magurele-Bucharest, Romania

Current address: Cankaya University, Faculty of Art and Science, Department of Mathematics and Computer Sciences, Ankara, Turkey

E-mail address: dumitru@cankaya.edu.tr 